\title{
On Predicting Roller Milling Performance V: Effect of Moisture Content on the Particle Size Distribution from First Break Milling of Wheat
}

\author{
Chaoying Fang and Grant M Campbell*
}

\author{
Satake Centre for Grain Process Engineering, Department of Chemical Engineering, \\ UMIST, Manchester, M60 1QD, UK
}

Received 16 October 2001

\begin{abstract}
Extended breakage functions incorporating both single kernel size and moisture content were determined for First Break milling of hard and soft wheats under a sharp-to-sharp disposition. A moisture correction function was constructed by milling narrow size fractions of wheat tempered to different moisture contents, and subtracting the breakage function at a base moisture content of $16 \%$ from that at other moisture contents. The effect of adding moisture was to change an initially inverted U-shaped distribution at low moisture contents to a linear distribution at $16 \%$ moisture, then to a U-shaped distribution at higher moisture contents, reminiscent of the particle size distributions produced by dull-to-dull milling. The extended breakage functions were used to predict milling of unseparated feed samples at different roll gaps and moisture contents. In addition, mixtures of hard wheat at $14 \%$ moisture and soft wheat at $20 \%$ moisture, mixed in different proportions, were milled and the resulting particle size distributions compared with predictions. Excellent predictions were obtained in all cases. This confirms the independent breakage of kernels during First Break milling, and demonstrates the potential of the breakage function approach for interpreting single kernel data in terms of predictions of milling performance.

(C) 2003 Published by Elsevier Science Ltd
\end{abstract}

Keywords: first break milling, moisture, particle size distribution, breakage equation.

\section{INTRODUCTION}

Previous papers in this series have introduced the breakage equation that describes roller milling of wheat grains $^{1}$; derived the form of the breakage function that describes the particle size distribution resulting from breakage of an individual wheat kernel as a function of kernel size and roll gap ${ }^{2}$; demonstrated that this form of the breakage function applies for a wide range of wheat varieties ${ }^{3}$, and investigated the effect of roll disposition on the breakage function ${ }^{4}$. This work has demonstrated

\footnotetext{
* Corresponding author. Tel: +44(0)1612004472; Fax: +44(0)161 200 4399; E-mail: g.campbell@umist.ac.uk.
}

that, knowing the form of the breakage function, the particle size distribution resulting from First Break milling of wheat can be predicted for any inlet size distribution and any roll gap. This allows the inlet and outlet streams from First Break roller milling to be linked mathematically, and forms a sound basis for predicting roller milling performance from measured distributions of single kernel parameters. The objective of the current work was to add moisture content to the breakage function, to allow the effect of moisture content on First Break milling of wheat to be predicted.

The three parts of the wheat kernel, bran, germ and endosperm, differ in relative toughness and friability, giving different breakage patterns on roller 
milling. These differences are exaggerated by adding water to the wheat prior to milling, in a process known as conditioning or tempering. Conditioning has five purposes $^{5-7}$ :

(i) to toughen the bran, reducing formation of bran powder;

(ii) to soften the endosperm, enhancing its millability and reducing the power consumed by the reduction rolls;

(iii) to facilitate separation of bran from endosperm, reducing the power consumption of the break rolls and consequently reducing evaporative losses;

(iv) to ensure easy and accurate sifting of stocks; and

(v) to ensure the endosperm moisture content is sufficient to give a final flour moisture content of around $14-15 \%$.

However, the amount of water added and the timescale over which it is allowed to penetrate into the kernel vary widely in practice, with no conditioning regime universally appropriate for all wheat types and milling systems. Typically soft wheats are conditioned to $15-15.5 \%$ moisture and hard wheats to $16-16 \cdot 5 \% \mathrm{wb}$.

Research into wheat conditioning has encompassed studies on wheat physical properties as affected by moisture content ${ }^{8-14}$; rates of water uptake $^{15,16}$ and moisture distribution in the grain ${ }^{17}$; the effect of moisture on breakage during roller milling ${ }^{18-20}$; comparison and evaluation of conditioning regimes ${ }^{21,22}$, including accelerated conditioning $^{23-25}$, and the effect of conditioning on flour properties and performance ${ }^{20,22,26-30}$. Conditioning studies have also been reported for other grains, including oats ${ }^{31}$, barley ${ }^{32}$ and sorghum ${ }^{33}$.

Zoerb and $\mathrm{Hall}^{8}$ concluded that moisture greatly affects mechanical properties of grains, and that all strength properties of grains decrease in magnitude with increasing moisture contents. However the nature and extent of the decrease differs between bran and endosperm. Glenn et al. ${ }^{11}$ showed that as the moisture content of wheat endosperm increases, the compressive strength, elasticity and energy to compressive failure all decrease, with hard wheats giving greater decreases. By contrast, the elasticity and plasticity of bran increase with increasing moisture content ${ }^{12,13}$. This renders the bran capable of deforming without breaking to a degree far beyond that of the endosperm. Thus, adding moisture to wheat prior to milling facilitates breakage of endosperm while making bran more resistant to breakage.

While numerous papers have investigated the effect of moisture on the mechanical properties of the components of the wheat kernel, fewer studies have quantified the effect on the particle size distribution resulting from milling. Hsieh et al. ${ }^{19}$ studied the effect of moisture content after tempering on the First Break release of a Canadian spring wheat. In their study break release was defined as the proportion by mass of broken particles smaller than $730 \mu \mathrm{m}$. They found that over the range of tempering moisture contents from 14.5 to $17.5 \%$, First Break release increased with increasing moisture content. This is as expected from the knowledge of the effect of moisture on endosperm properties, and confirms similar results by Anderson ${ }^{18}$. Dexter and Martin's ${ }^{20}$ results showed little influence of wheat moisture content over the range $10-25 \%$ on break release through $980 \mu \mathrm{m}$ from First and Second Break rolls, although break release from Third and Fourth Break rolls declined dramatically with increasing wheat moisture content.

In the present study the breakage function derived previously was extended to include the effect of moisture content in predictions of the particle size distribution resulting from First Break milling of wheat.

\section{THEORY}

The breakage equation for roller milling, expressed in its cumulative form, is

$$
P(x)=\int_{0}^{\infty} B(x, D) \rho_{1}(D) d D
$$

where $D$ is the size of a feed particle, $x$ is the size of an outlet particle, $\rho_{1}(D)$ is the probability density function describing the particle size distribution of the feed material, $P(x)$ is the proportion by mass of material smaller than $x$ in the outlet stream, and $B(x, D)$ is the cumulative breakage function describing the proportion of material smaller than $x$ produced on breakage of a feed particle initially of size $D$. In this work $D$ is measured as the third longest dimension (thickness) of a wheat kernel, while $x$ is measured as the sieve diameter of milled material. Equation (1) allows the particle size distribution resulting from milling a mixture of feed particle sizes to be predicted. 
Wheat kernels are heterogeneous in more than just size, a fact underlined by the introduction of the Single Kernel Characterisation System, which measures the distributions of mass, diameter, moisture content and hardness of kernels in a sample ${ }^{34,35}$. In principle equation (1) can be extended to encompass distributions of other kernel properties in the feed. The current paper aims to demonstrate this with respect to moisture content. Shellenberger ${ }^{6}$ notes that kernel moisture can vary by 5 percentage points within a sample both before and after conditioning, and that the time required for kernels differing in moisture content to approach equilibrium can be several months. Therefore although the average kernel moisture content might be optimal, some kernels are too wet and others too dry to give optimum flour yield. This variability in kernel moisture may be exacerbated by the practice of mixing wet and dry wheat together to preserve wet wheat and add moisture to dry wheat ${ }^{36}$.

Equation (1) can be extended to account for moisture content distribution as follows:

$$
P(x)=\int_{0}^{m_{\max }} \int_{0}^{\infty} B(x, D, m) \rho_{1}(D) \rho_{2}(m) d D d m
$$

where $m$ is the moisture content of an individual kernel, and $\rho_{2}(m)$ is the probability density function describing the distribution of individual kernel moisture contents in the sample. Equation (2) assumes that the size distribution and moisture distribution in a sample are independent, i.e. that large kernels have the same moisture distribution as small kernels (or, equivalently, that wetter kernels have the same size distribution as drier kernels). Thus, if the extended cumulative breakage function $B(x, D, m)$ is known, then the outlet particle size distribution could be predicted for any size distribution and moisture distribution of a given wheat sample.

If it is assumed that the effect of moisture content on breakage is approximately independent of kernel size, then the cumulative breakage function can be simplified by separating it into two components, the cumulative breakage function at a nominal moisture content $m_{0}$ (say 16\%), and a correction term to account for the variation in breakage that occurs at moisture contents other than $16 \%$ :

$$
B(x, D, m) \approx B(x, D)_{m_{0}}+K\left(x, m-m_{0}\right)
$$

Then equation (2) becomes

$$
\begin{aligned}
P(x)_{m}= & \int_{0}^{m_{\max }} \int_{0}^{\infty}\left[B(x, D)_{m_{0}}+K\left(x, m-m_{0}\right)\right] \\
& \quad \times \rho_{1}(D) \rho_{2}(m) d D d m \\
= & P(x)_{m_{0}}+\int_{0}^{m_{\max }} K\left(x, m-m_{0}\right) \rho_{2}(m) d m
\end{aligned}
$$

In equation (4), the cumulative breakage function $B(x, D)_{m_{0}}$ can be found by milling narrow size fractions of wheat conditioned to $16 \%$, as described previously $^{2-4}$. The moisture correction function, $K\left(x, m-m_{0}\right)$ can be found similarly by milling samples tempered to different moisture contents and subtracting the proportion of material smaller than size $x$ at the base moisture content $m_{0}$ from the corresponding proportion at moisture contents $m$.

If the samples prepared to different moisture contents are also separated into narrow size fractions, the assumption that the effect of moisture content on breakage is approximately independent of kernel size could be tested. In practice, previous work has demonstrated that the relevant parameter related to size is the milling ratio, $G / D$, where $G$ is the roll gap ${ }^{2-4}$. Milling at different roll gaps is equivalent to milling different size fractions. In this paper combinations of size fractions and roll gaps were used to cover a range of milling ratios.

If the moisture distribution in a sample is very narrow, such that $\rho_{2}(\mathrm{~m})$ approximates a Dirac delta function, or if only the average moisture content is available, then equation (4) can be used in the form

$$
P(x)_{m}=P(x)_{m_{0}}+K\left(x, m-m_{0}\right)
$$

where $m$ is the average moisture content of kernels in the sample. In its non-cumulative form, equation (5) becomes

$$
\rho_{2}(x)_{m}=\rho_{2}(x)+k\left(x, m-m_{0}\right)
$$

where

$$
k\left(x, m-m_{0}\right)=\frac{d}{d x} K\left(x, m-m_{0}\right)
$$




\section{MATERIALS AND METHODS}

\section{Construction of the moisture correction function}

Hereward, a hard wheat (bulk density $79.4 \mathrm{~kg} / \mathrm{hl}$, protein content $14.6 \% \mathrm{db}$, moisture content $14 \cdot 16 \% \mathrm{wb}$ ), and Consort, a soft wheat (bulk density $74.2 \mathrm{~kg} / \mathrm{hl}$, protein content $10.63 \% \mathrm{db}$, moisture content $13.97 \% \mathrm{wb})$, both from the $1999 \mathrm{UK}$ harvest, were separated by thickness into narrow size fractions as described previously ${ }^{2,4}$. Table I gives the size fractions and roll gaps used to give a range of milling ratios.

The separated fractions were then dried for $24 \mathrm{~h}$ at $40^{\circ} \mathrm{C}$ in an incubator, after which the moisture content of the Hereward had reduced to $8.8 \%$ and that of the Consort to $9 \cdot 3 \%$, as measured by an oven drying method ${ }^{37}$. Each dried fraction was then separated into seven batches, to which were added different amounts of water using a pipette, to give nominal moisture contents of 10,12, 14, 16, 18 and $20 \%$ plus the original dried moisture content for each variety. Samples were left overnight to temper and then separated into five samples of $100 \mathrm{~g}$ each, one for each milling ratio listed in Table I. A total of 70 samples were prepared. Each $100 \mathrm{~g}$ sample was then milled at the appropriate milling ratio on the Satake STR-100 test roller mill using 10.5 flutes per inch $(4.13$ flutes per $\mathrm{cm})$ First Break rolls operated under a sharp-to-sharp disposition. Milling and subsequent sieve analysis were performed as described previously ${ }^{4,38}$.

The mean diameter of each fraction following moisture addition and tempering was measured using the Perten SKCS (Perten Instruments AB, Sweden) to ensure that the addition of moisture had not altered the size of grains substantially.

Table I Size fraction and roll gap combinations used to mill wheat samples at a range of milling ratios

\begin{tabular}{lcc}
\hline Size $D(\mathrm{~mm})$ & Roll gap $G(\mathrm{~mm})$ & $G / D$ \\
\hline Hereward & & \\
$3 \cdot 00-3 \cdot 25$ & $0 \cdot 3$ & $0 \cdot 096$ \\
& $0 \cdot 4$ & $0 \cdot 128$ \\
& $0 \cdot 5$ & $0 \cdot 160$ \\
& $0 \cdot 6$ & $0 \cdot 192$ \\
Consort & $0 \cdot 7$ & $0 \cdot 224$ \\
$3 \cdot 00-3 \cdot 25$ & & \\
$2 \cdot 75-3 \cdot 00$ & $0 \cdot 3$ & $0 \cdot 096$ \\
& $0 \cdot 4$ & $0 \cdot 139$ \\
$2 \cdot 50-2 \cdot 75$ & $0 \cdot 5$ & $0 \cdot 174$ \\
& $0 \cdot 6$ & $0 \cdot 229$ \\
& $0 \cdot 7$ & $0 \cdot 267$ \\
\hline
\end{tabular}

The difference in diameter between the lowest and highest moisture contents was less than $2 \%$ and could be neglected compared with the intervals between the different milling ratios used.

The moisture correction function was calculated by subtracting the percentage of material by mass smaller than $x$ at a moisture content of $16 \%$ from the corresponding percentage at other moisture contents. The breakage function reported previously ${ }^{4}$ for the two wheat varieties milled at $16 \%$ moisture was used as the base from which the moisture correction function was derived, as it had been evaluated using a greater number of samples than was used here for each moisture content and was thus more accurate.

\section{Prediction of milling of whole wheat samples and of mixtures of wheats}

To enable evaluation of the combined breakage and moisture correction functions in terms of prediction, two further sets of milling trials were performed. In the first, native (unseparated) samples of Hereward and Consort were prepared to different moisture contents $(10,12,14,16,18$ and 20\%) and milled at roll gaps of 0.4 and $0.6 \mathrm{~mm}$. The moisture distribution of each sample was measured using the SKCS. Figure 1 shows the measured moisture distributions for each sample. Note that the SKCS is capable of measuring moisture content accurately only up to $15 \%{ }^{39}$. Nevertheless, the measured moisture distributions were used in equation (4) to predict the output particle size distribution for each sample at each milling ratio.

In the second trial, samples of Hereward at 14\% moisture and Consort at 20\% moisture were mixed in the ratios $100: 0,75: 25,50: 50,25: 75$ and $0: 100$ Hereward:Consort, and milled at a roll gap of $0.6 \mathrm{~mm}$, to demonstrate the ability to predict milling of mixtures of different wheats. For both trials three replicate $100 \mathrm{~g}$ samples were milled for each condition and compared with predictions based on proportional addition of the individual predictions for each variety.

\section{RESULTS AND DISCUSSION \\ Construction of the moisture correction function}

Figure 2 shows the proportion of milled material smaller than a given sieve aperture $x$ as a function 


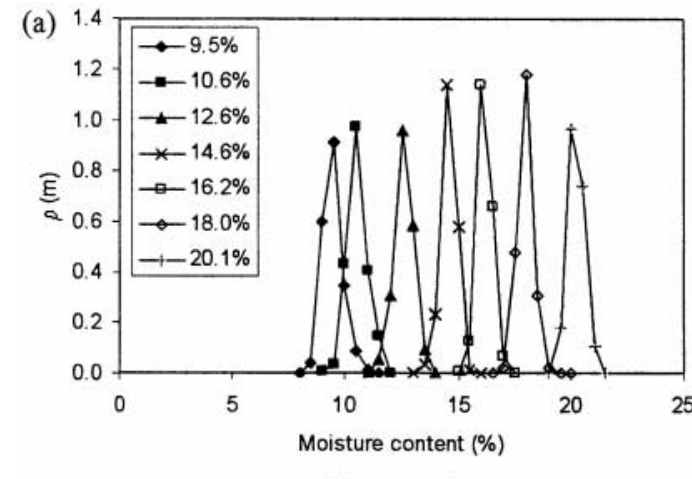

Hereward

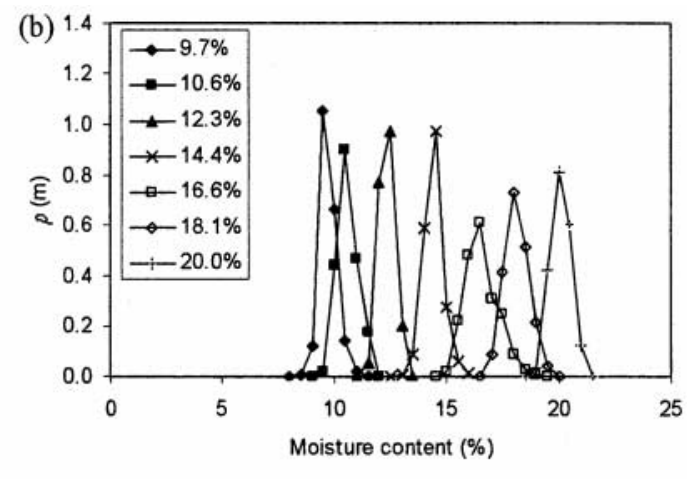

Consort

Figure 1 Moisture distributions of (a) Hereward and (b) Consort after conditioning.

of milling ratio for each value of $x$ and for each moisture content, for the Hereward sample. The approximate parallelism of the lines for different moisture contents at each value of $x$ justifies the simplifying assumption that the effect of moisture content on breakage is independent of milling ratio. Similar results (not shown) were obtained for the Consort. The function $B(x, D, m)$ was fitted to the data using Microsoft Excel (Microsoft Corporation, USA). From these curves, the average $K(x, D, m-$ $16 \%$ ) was calculated as

$$
\begin{aligned}
K(x, m-16 \%) & =\frac{1}{\mathcal{N}} \sum_{D} K(x, D, m-16 \%) \\
& =\frac{1}{\mathcal{N}} \sum_{D} B(x, D, m)-B(x, D, 16 \%)
\end{aligned}
$$

where $\mathcal{N}$ is the number of milling ratios at which samples were milled. An equation cubic in $x$ and quadratic in $(m-16 \%)$ was fitted to the data:

$$
\begin{aligned}
K(m-16 \%, x) & =\left(a_{1}+b_{1} x+c_{1} x^{2}+d_{1} x^{3}\right)(m-16 \%) \\
& +\left(a_{2}+b_{2} x+c_{2} x^{2}+d_{2} x^{3}\right)(m-16 \%)^{2}
\end{aligned}
$$

Table II lists the coefficients of equation (9) fitted for both wheat samples, along with the coefficients of determination, which indicate a good description of the data for both samples.

The derivative of equation (9) with respect to $x$ is a quadratic in $x$, indicating that the effect of changing the moisture content is to change the initially linear breakage function obtained under sharp-to-sharp
Table II Coefficients of the moisture correction function for Hereward and Consort

\begin{tabular}{lrrrr}
\hline & \multicolumn{1}{c}{$a$} & \multicolumn{1}{c}{$b$} & $c$ & \multicolumn{1}{c}{$d$} \\
\hline Hereward & & & & \\
1 & $-72 \cdot 3$ & $0 \cdot 652$ & $-8.09 \mathrm{E}-04$ & $2 \cdot 39 \mathrm{E}-07$ \\
2 & 1702 & $-12 \cdot 818$ & $7 \cdot 56 \mathrm{E}-03$ & $-9 \cdot 7 \mathrm{E}-07$ \\
$R^{2}$ & & & 0.933 & \\
Consort & & & & \\
1 & $-40 \cdot 7$ & 0.406 & $-7 \cdot 03 \mathrm{E}-04$ & $2 \cdot 27 \mathrm{E}-07$ \\
2 & $93 \cdot 6$ & $-5 \cdot 685$ & $3.70 \mathrm{E}-03$ & $-7 \cdot 6 \mathrm{E}-07$ \\
$R^{2}$ & & & 0.952 & \\
\hline
\end{tabular}

milling to the more U-shaped distribution found for other dispositions.

Figures 3 and 4 show the particle size distributions (non-cumulative) obtained on milling of fractions at different moisture contents and milling ratios for Hereward and Consort, respectively. These figures also show the actual average moisture contents achieved, as measured by the SKCS. As indicated above, the effect of moisture content is to change the approximately linear particle size distributions observed under sharp-to-sharp milling of samples at $16 \%$ moisture to more U-shaped distributions. At low moisture contents the $\mathrm{U}$ is inverted; fewer particles are generated at the large and small ends of the distribution, and more particles are seen in the mid-size range, pushing the centre of the distribution up. As moisture content increases, the $\mathrm{U}$ inverts, such that at high moisture contents large numbers of smaller and larger particles are seen, with fewer in the mid-size range, reminiscent of the shapes of distributions seen under dull-to-dull milling ${ }^{4}$. This effect is most clearly demonstrated by the data in Figure 4(d) and (e), for Consort milled at milling ratios of $0 \cdot 192$ and $0 \cdot 224$. 

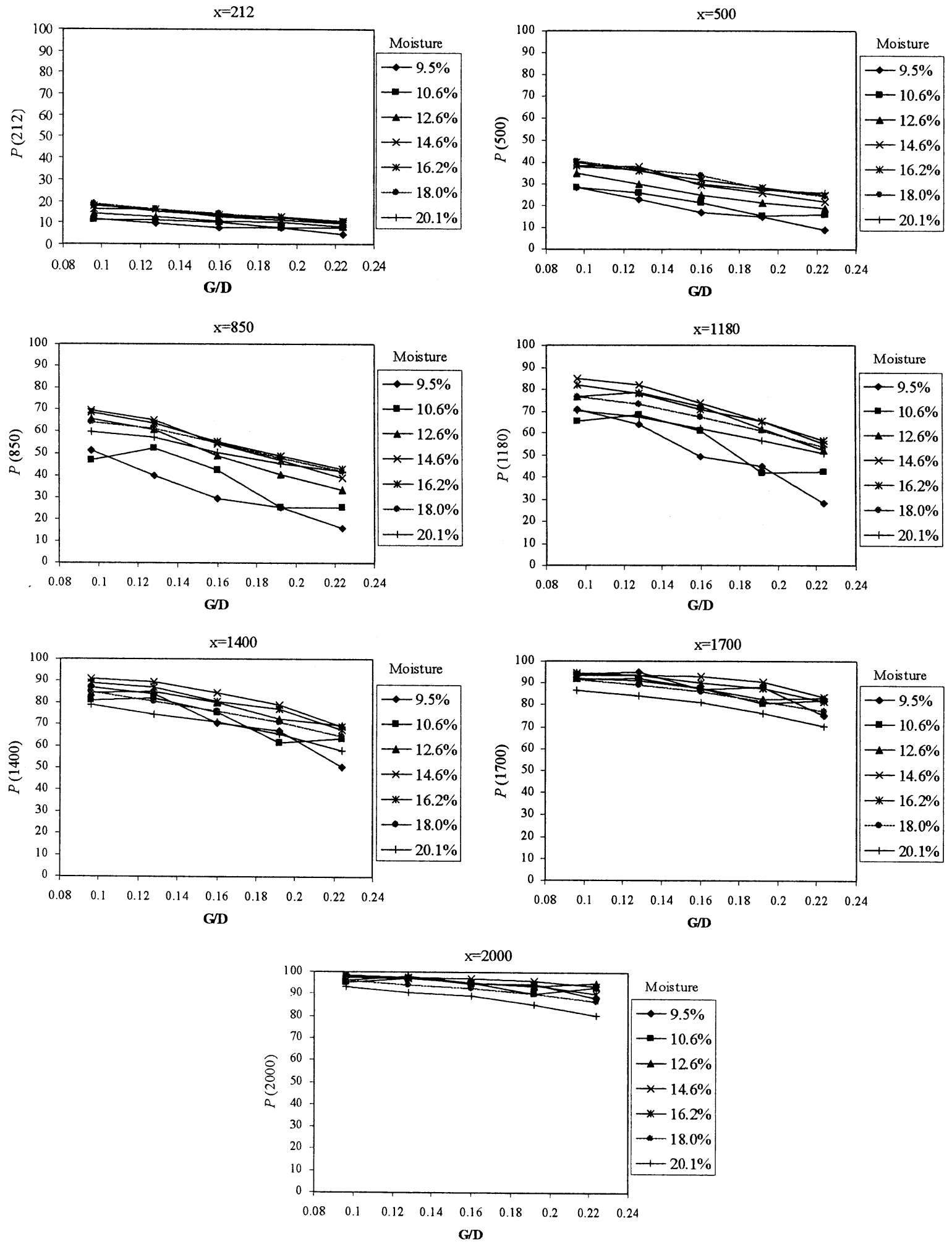

Figure 2 Proportion of milled material smaller than sieve aperture size $x$ vs milling ratio for Hereward (hard) wheat fractions tempered to different moisture contents. 


\section{Prediction of milling of whole wheat samples and of mixtures of wheats}

The moisture correction functions derived above were used to predict the milling of native (unseparated) samples. Figures 5 and 6 compare predicted cumulative particle size distributions with experimental data for both wheats tempered to different moisture contents and milled at roll gaps of 0.4 and $0.6 \mathrm{~mm}$. Clearly the agreement is excellent, as indicated by the $R^{2}$ values shown in the figure captions. Figure 7 similarly shows the predictions (a)

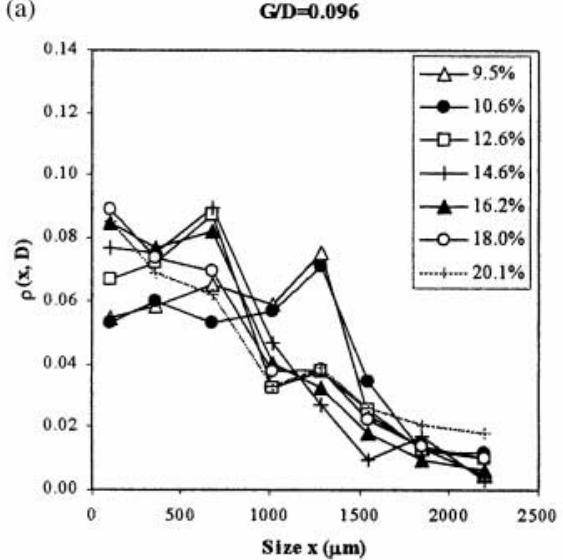

(c)

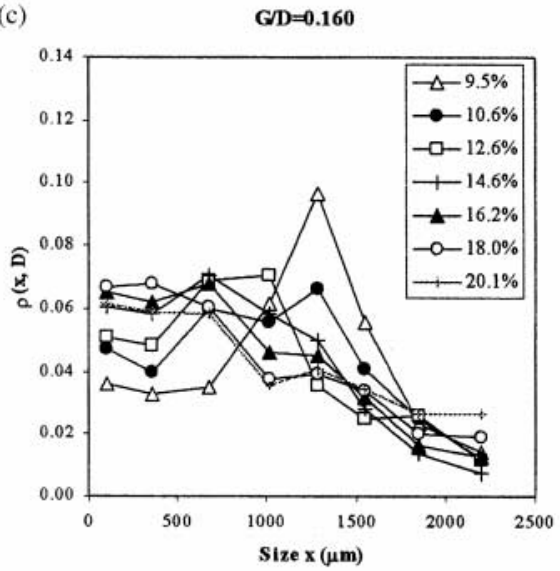

(b)

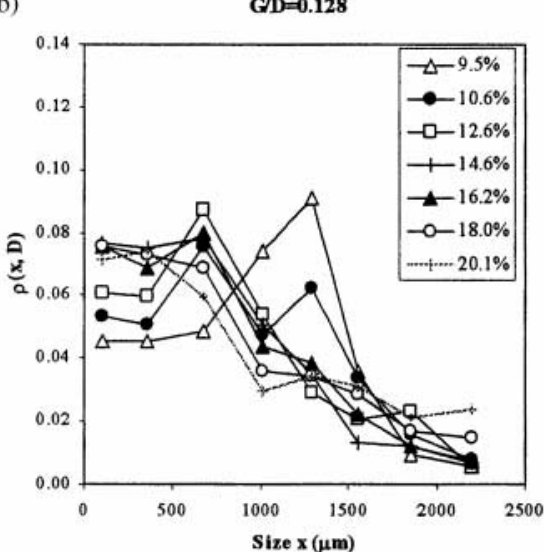

(d)

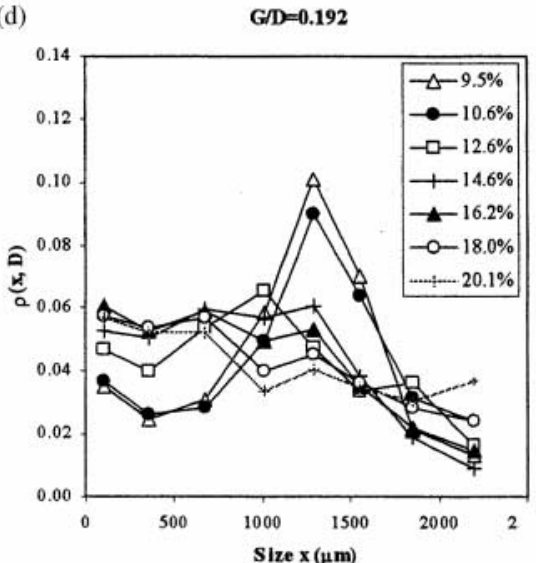

(e)

$\mathrm{G} / \mathrm{D}=\mathbf{0 . 2 2 4}$

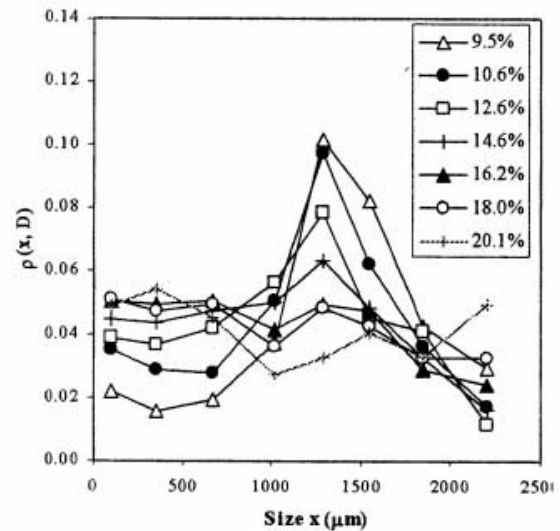

Figure 3 Size distribution of stocks from First Break milling at different milling ratios of Hereward (hard) wheat tempered to different moisture contents. 

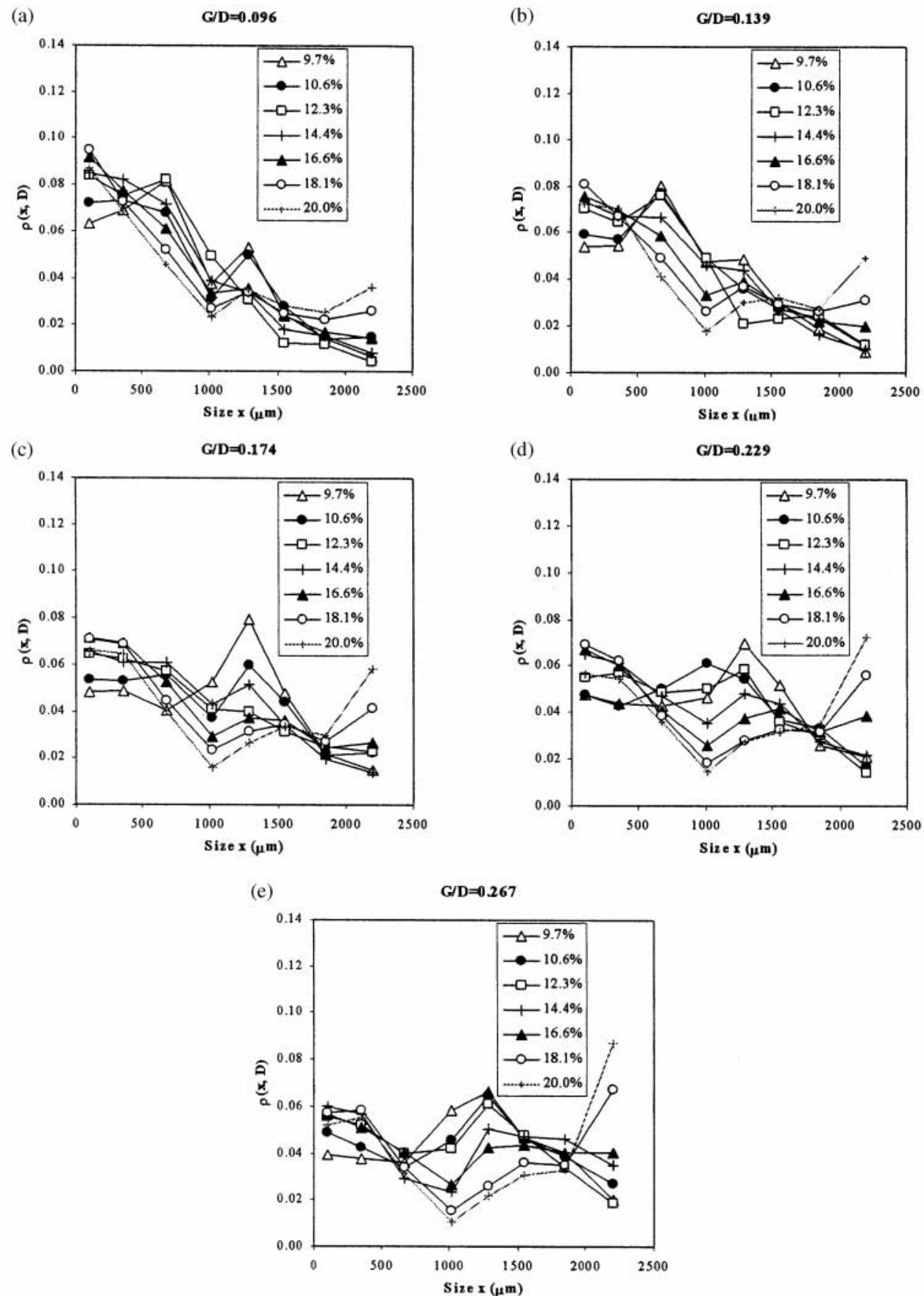

Figure 4 Size distribution of stocks from First Break milling at different milling ratios of Consort (soft) wheat tempered to different moisture contents.

when Hereward at 14\% moisture and Consort at $20 \%$ moisture are mixed in different ratios and milled together. The excellent agreement confirms that the breakage function approach is able to predict milling of mixtures of wheats, and supports the underlying assumption that kernels mill independently during First Break roller milling. While it is understood that this is an unlikely mixture to be created in practice, it demonstrates the accuracy and potential of the breakage function approach. 

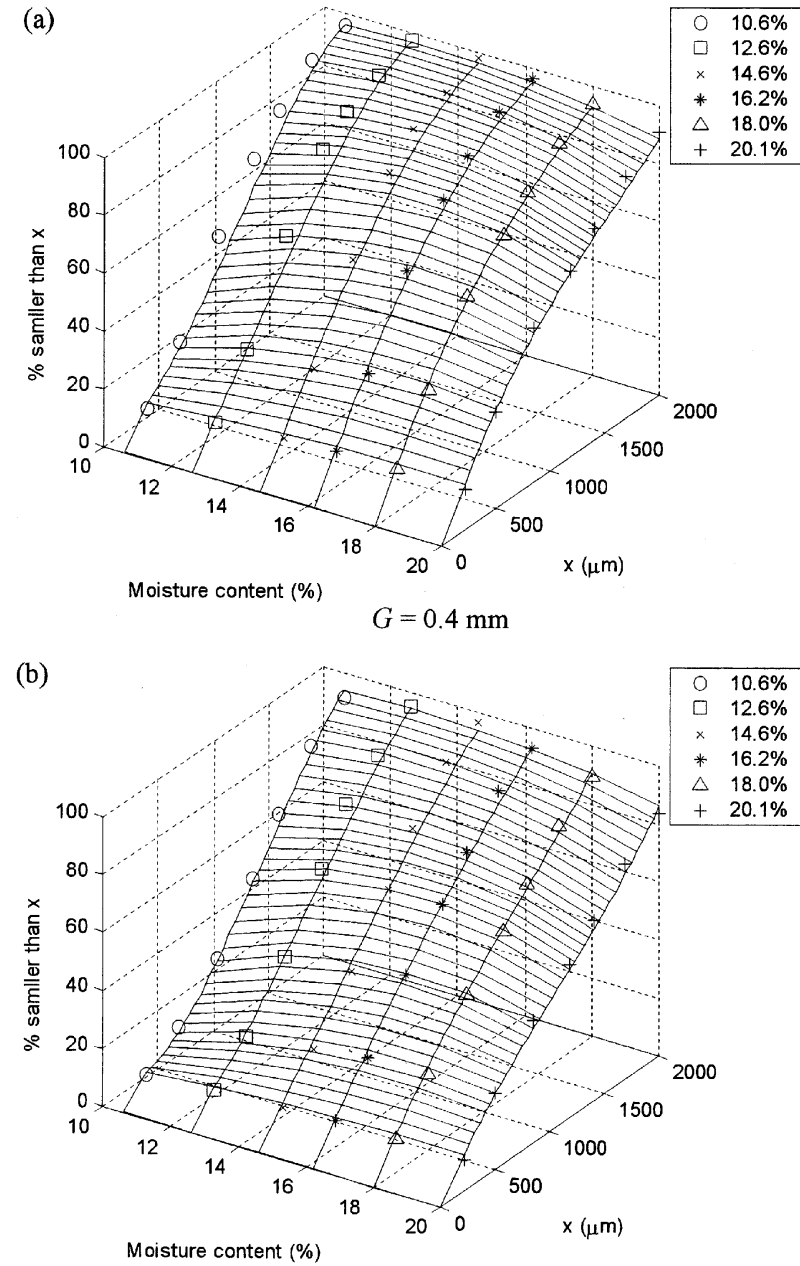

$G=0.6 \mathrm{~mm}$

Figure 5 Comparison of predicted and experimental particle size distributions from First Break milling of Hereward wheat at roll gaps of (a) $0.4 \mathrm{~mm}\left(R^{2}=0.985\right)$; and (b) $0.6 \mathrm{~mm}\left(R^{2}=0.992\right)$. Solid lines are predictions based on Equations (5) and (9), and symbols are experimental data averaged from three replicate samples.

(Note that predictions based on Equation (5), the simplified equation that considers only the average moisture content and not its distribution, gave virtually identical predictions. This is because of the narrow distribution of single kernel moisture contents actually achieved in these samples, as indicated by Figure 1. Note too that good predictions are obtained despite some moisture contents being above the upper limit of $15 \%$ for which the SKCS is considered to give accurate moisture results.)

Interestingly, the effect of adding moisture to wheat is similar to the effect of milling under a dull-to-dull disposition. Both favour the creation of large and small particles with few in the mid-size
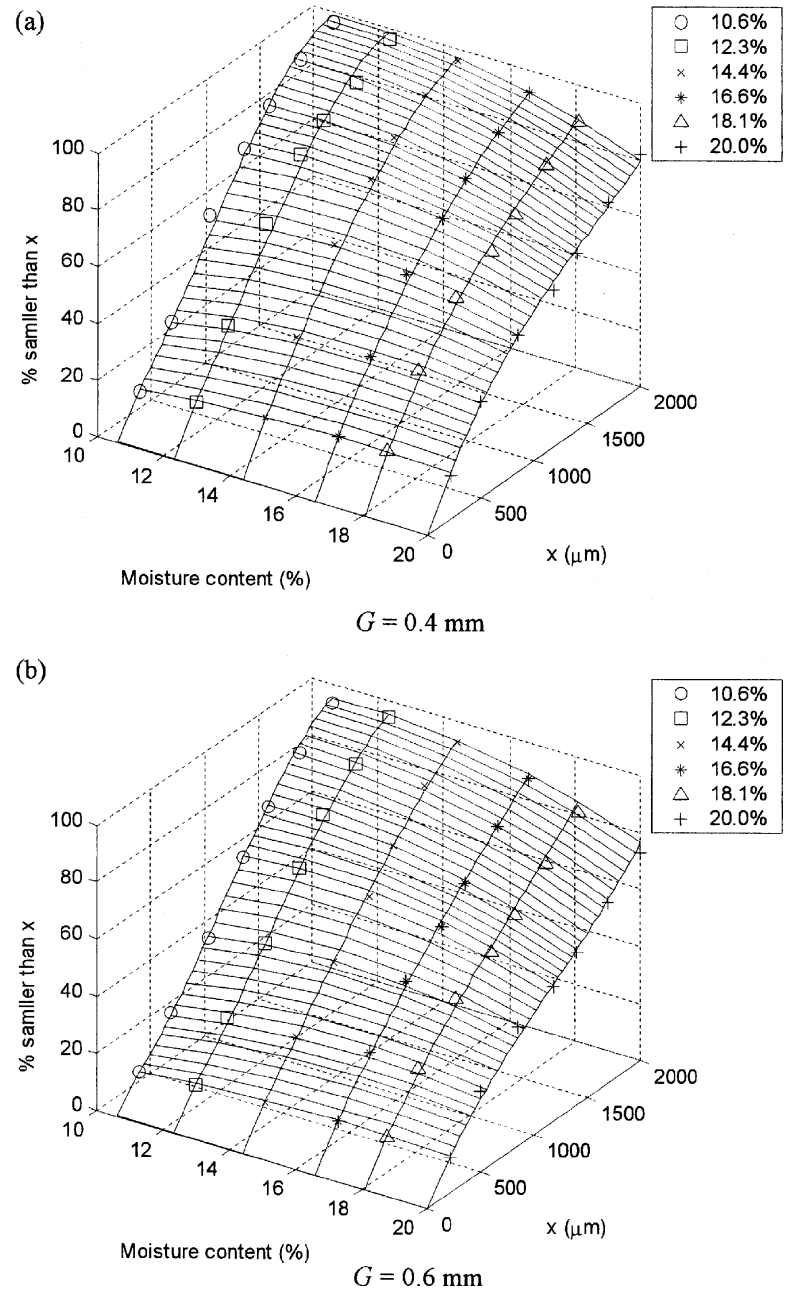

Figure 6 Comparison of predicted and experimental particle size distributions from First Break milling of Consort wheat at roll gaps of (a) $0.4 \mathrm{~mm}\left(R^{2}=0.991\right)$; and (b) $0.6 \mathrm{~mm}\left(R^{2}=0.996\right)$. Solid lines are predictions and symbols are experimental data.

range, thus facilitating separation of the large branny particles from the smaller endosperm particles. This undoubtedly explains in part the preferred practice of millers to add moisture to wheat and then mill it under a dull-to-dull disposition at First Break.

\section{CONCLUSIONS}

Extended breakage functions have been constructed to describe First Break milling of wheat kernels as functions of single kernel size and moisture content. The ability of these functions to predict milling of polydisperse feeds at different moisture contents and of mixtures of different wheats has been demonstrated. This confirms the independent breakage of wheat kernels during First Break milling, and 


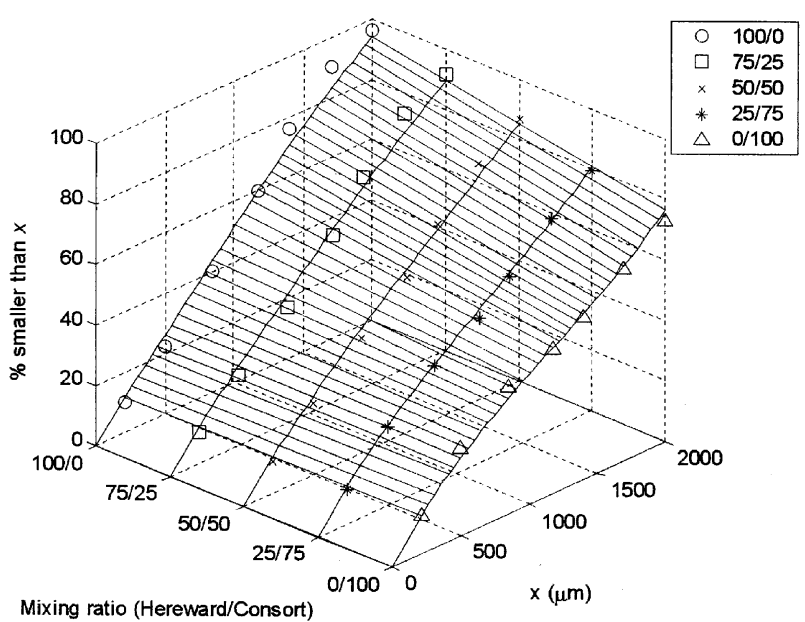

Figure 7 Comparison of predicted and experimental particle size distributions from First Break milling of mixtures of Hereward at $14 \%$ moistures and Consort at $20 \%$ moisture at a roll gap of $0.6 \mathrm{~mm}\left(R^{2}=0.987\right)$. Solid lines are predictions and symbols are experimental data.

demonstrates the potential of the breakage function approach for linking distributions of single kernel measurements to milling performance. Future work needs to identify other appropriate single kernel properties such as hardness and incorporate them into a comprehensive breakage function that would allow prediction of milling performance directly from single kernel measurements.

\section{Acknowledgements}

This work was funded by the Engineering and Physical Sciences Research Council (EPSRC, Grant No. GR/ M49939). The support of the Satake Corporation of Japan is gratefully acknowledged.

\section{REFERENCES}

1. Campbell, G.M. and Webb, C. On predicting roller milling performance. I. The breakage equation. Powder Technology 115 (2001) 234-242.

2. Campbell, G.M., Bunn, P.J., Webb, C. and Hook, S.C.W. On predicting roller milling performance. II. The breakage function. Powder Technology 115 (2001) 243-255.

3. Bunn, P.J., Campbell, G.M., Fang, C. and Hook, S.C.W. On predicting roller milling performance. III. The particle size distribution from roller milling of various wheats using fluted rolls. 6th World Chemical Engineering Congress (2001) Melbourne, Australia.

4. Fang, C. and Campbell, G.M. On predicting roller milling performance. IV. Effect of roll disposition on the particle size distribution from First Break milling of wheat. Fournal of Cereal Science (accepted).

5. Lockwood, J.F. Flour Milling, The Northern Publishing Co. Ltd., London, UK (1945).
6. Shellenberger, J.A. Advances in milling technology. In 'Advances in Cereal Science and Technology', Vol 3, (Y. Pomeranz ed.), American Association of Cereal Chemists, St. Paul, Minnesota, USA (1980) pp. 227-270.

7. Sugden, T.D. Wheat flour milling, Part 1. In 'Cereals and Cereal Products: Chemistry and Technology', (D. A. V. Dendy and B. J. Dobraszczyk eds), Aspen Publishers Inc., Maryland, USA (2001) pp 140-172.

8. Zoerb, G.C. and Hall, C.W. Some mechanical and rheological properties of grains. Fournal of Agricultural Engineering Research 5 (1960) 83-93.

9. Yamazaki, W.T. and Donelson, J.R. Kernel hardness of some US wheats. Cereal Chemistry 60 (1983) 344-350.

10. Lawton, J.W. and Faubion, J.M. Measuring kernel hardness using the tangential abrasive dehulling device. Cereal Chemistry 66 (1989) 519-524.

11. Glenn, G.M., Younce, F.L. and Pitts, M.J. Fundamental physical properties characterizing the hardness of wheat endosperm. Fournal of Cereal Science 13 (1991) 179-194.

12. Glenn, G.M. and Johnston, R.K. Moisture-dependent changes in the mechanical properties of isolated wheat bran. Fournal of Cereal Science 15 (1992) 223-236.

13. Mabille, F., Gril, J. and Abecassis, J. Mechanical properties of wheat seed coats. Cereal Chemistry 78 (2001) 231-235.

14. Haddad, Y., Mabille, F., Mermet, A., Abecassis, J. and Benet, J.C. Rheological properties of wheat endosperm with a view on grinding behaviour. Powder Technology 105 (1999) 89-94.

15. Stenvert, N.L. and Kingswood, K. An autoradiographic demonstration of the penetration of water into wheat during tempering. Cereal Chemistry 53 (1976) 141-149.

16. Stenvert, N.L. and Kingswood, K. Factors influencing the rate of moisture penetration into wheat during tempering. Cereal Chemistry 54 (1977) 627-637.

17. Seckinger, H.L., Wolf, M.J. and Dimler, R.J. A micro method for determining moisture distribution in wheat kernels, based on iodine staining. Cereal Chemistry 41 (1964) 80-87.

18. Anderson, J.E. What water does to wheat in tempering. Association of Operative Millers Bulletin. May (1937) 794-797.

19. Hsieh, F.H., Martin, D.G., Black, H.C. and Tipples, K.H. Some factors affecting the First break grinding of Canadian wheat. Cereal Chemistry 57 (1980) 217-213.

20. Dexter, J.E. and Martin, D.E. Influence de la teneur en eau sur le comportement en moutre d'un blé CWRS. Industries des Céréales 81 (1993) 5-13.

21. Sibbitt, L.D., Classon, D.H. and Harris, R.H. Improved tempering and modified milling techniques for small samples of wheat. Cereal Chemistry 37 (1960) 398-404.

22. Pomeranz, Y., Bolte, L.C., Finney, K.F. and Shogren, M.D. Effect of variations in tempering on micromilling of hard winter wheat. Cereal Chemistry 62 (1985) 47-50.

23. Doty, N.C. and Baker, C.W. Microwave conditioning of hard red spring wheat. I. Effects of wide power range on flour and bread quality. Cereal Chemistry 54 (1977) 717-727.

24. Finney, K.F. and Bolte, L.C. Experimental micromilling: Reduction of tempering time of wheat from $18-24 \mathrm{~h}$ to 30 min. Cereal Chemistry 62 (1985) 454-458.

25. Finney, P.L. and Andrews, L. A 30-minute conditioning method for micro-, intermediate- and large-scale experimental milling of soft red winter wheat. Cereal Chemistry 63 (1986) 18-21. 
26. Sullivan, B., Anderson, M.L. and Goldstein, A.M. The determination of starch damage of flour. Cereal Chemistry 39 (1962) 155-167.

27. Wolf, M.J., Kwolek, W.F. and McCarthy, J.R. Quantitative microscopic evaluation of endosperm breakdown in conditioned hard red winter wheat. Cereal Chemistry 43 (1966) 43-61.

28. Kathuria, D.K. and Sidhu, J.S. Indian durum wheats. I. Effect of conditioning treatments on the milling quality and composition of semolina. Cereal Chemistry 61 (1984) 460-462.

29. Kathuria, D.K. and Sidhu, J.S. Indian durum wheats. II. Effect of conditioning treatments on the quality of spaghettis. Cereal Chemistry 61 (1984) 463-465.

30. Gobin, P., Duviau, M.P., Wong, J.H., Buchanan, B.B. and Kobrehel, K. Change in sulfhydryl-disulfide status of wheat proteins during conditioning and milling. Cereal Chemistry 73 (1996) 495-498.

31. Doehlert, D.C. and Moore, W.R. Composition of oat bran and flour prepared by three different mechanisms of dry milling. Cereal Chemistry 74 (1997) 403-406.

32. Bhatty, R.S. Milling of regular and waxy starch hull-less barleys for the production of bran and flour. Cereal Chemistry 74 (1997) 693-699.

33. Suroso, J., Flores, R.A. and Boyer, J.E. Scarification and degermination of sorghum for grits production: effects of hybrid and conditioning. Cereal Chemistry 77 (2000) 808-815.

34. Martin, C., Rousser, R. and Brabec, D.L. Development of a single kernel wheat characterisation system. Transactions of the American Society of Agricultural Engineers 36 (1993) 1399-1404.

35. Osborne, B.G. Wheat Flour Milling, Part 2. In 'Cereals and Cereal Products: Chemistry and Technology' (D. A. V. Dendy and B. J. Dobraszczyk, eds), Aspen Publishers Inc., Maryland, USA (2001) pp 172-181.

36. Bradbury, D., Hubbard, J.E., MacMasters, M.M. and Senti, F.R. 'Conditioning wheat for milling. A survey of the literature'. Agricultural Research Service, United States Department of Agriculture, Washington, D.C. (1960).

37. American Association of Cereal Chemists. Method 44-15A. In 'Approved Methods of the American Association of Cereal Chemists, 9th edition'. American Association of Cereal Chemists, St Paul, Minnesota, USA (1995).

38. Fang, C.Y. and Campbell, G.M. Effect of roll fluting disposition and roll gap on the breakage of wheat kernels during First Break roller milling. Cereal Chemistry 79 (2002) 518-522.

39. Single Kernel Characterization System 4100 Manual, Perten Instruments AB, Sweden, (1996). 